\title{
Escape from a chaotic attractor with fractal basin boundaries
}

\author{
Alexander N. Silchenko ${ }^{a}$, Stefano Beri ${ }^{a}$, Dmitry G. Luchinsky ${ }^{a}$, Peter V. E. McClintock ${ }^{a}$ \\ ${ }^{a}$ Department of Physics, Lancaster University, Lancaster LA1 4YB, UK
}

\begin{abstract}
We study fluctuational transitions between two co-existing chaotic attractors separated by a fractal basin boundary in a discrete dynamical system. It is shown that the mechanism of fluctuational transition through a fractal boundary is generic, and determined by a hierarchy of homoclinic original saddles. The most probable escape path from a chaotic attractor to the fractal boundary is found using both statistical analyses of fluctuational trajectories and the Hamiltonian theory of fluctuations.
\end{abstract}

Keywords: Fractal basin boundaries, noise-induced escape, homoclinic points

\section{INTRODUCTION}

The stability of nonlinear multistable chaotic systems in the presence of noise is of great importance in relation to a number of practical applications, and of obvious interdisciplinary interest. So also are methods for steering transitions between the co-existing chaotic attractors (CAs) in such systems. Quite generally, there are two distinct reasons why unpredictable and complex behavior may occur in a dynamical system. The first of these is the presence of limit sets with complex geometrical structure in phase space, and the second is complex structure of the basins of attraction, which may be fractal. ${ }^{5,6}$ Understanding the mechanism of fluctuational escape from a chaotic attractor through a fractal basin boundary (FBB) represents one of the most challenging unsolved problems in fluctuation theory $\cdot{ }^{1-4}$ Its complexity underlies the fact that we have hitherto lacked an answer to the central question: Does there exist a generic mechanism of fluctuational transition through an FBB? More specifically, it has been unclear: (i) whether boundary conditions could be found both on the CA and on the FBB; (ii) whether there is a unique escape path from the CA to the FBB; (iii) whether this path can be determined using the Hamiltonian theory of fluctuations; (iv) whether the transition through the FBB itself involves any deterministic structure; and (v) what is the effect of the noise intensity? If general features of the transition through the FBB do exist, a knowledge of them could considerably simplify analysis of both the stability and control of chaotic dynamical systems.

A promising approach to the solution of this problem is based on the analysis of fluctuations for very small noise intensity. In this limit, a stochastic dynamical system fluctuates to remote states along certain most probable deterministic paths, ${ }^{7-11}$ corresponding to rays in the WKB-like asymptotic solution of the FokkerPlanck equation. ${ }^{12}$ The possibility of extending such an approach to chaotic continuous and discrete systems was established earlier. ${ }^{1-4}$ It was shown also, that the presence of homoclinic tangencies, which are the reason for fractalization of the basins, causes a decrease in the activation energy. ${ }^{13}$

In this paper we show that a generic mechanism of fluctuational transitions between co-existing CAs separated by the FBB does exist, and that it is determined by a hierarchy of homoclinic original saddles forming the homoclinic structure of the FBB. In Sec. 2, we introduce a generic discrete model and describe its dynamics briefly. Section 3 deals with the determination of the boundary conditions on both the FBB and the CA. It is shown in Sec. 4 that there is a unique most probable escape path (MPEP) from the CA that approaches an accessible orbit on the fractal boundary. The MPEP is found using both a statistical analysis of fluctuational trajectories and the Hamiltonian theory of fluctuations. The effect of noise on the structure of escape paths inside the fractal basin is briefly analyzed. Our conclusions are presented in Sec. 5.

Correspondence to A.N.S. please. E-mail: a.silchenko@lancaster.ac.uk 


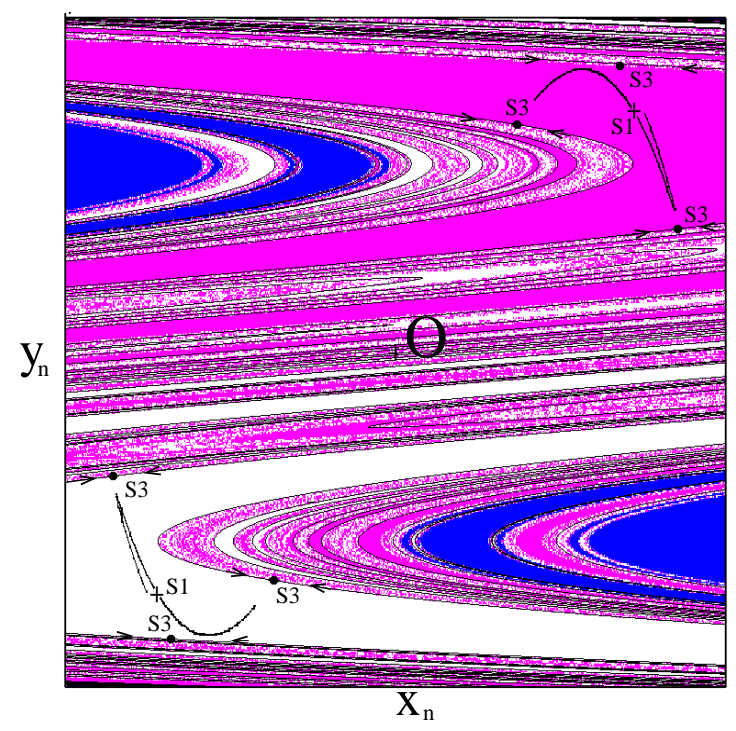

Figure 1. The co-existing chaotic attractors (full black curves); their basins of attraction are portrayed in grey and white respectively. The accessible boundary saddle points of period 3 are shown by as filled black circles and labelled S3. Their stable manifolds are indicated by solid black lines. The saddle pints of period 1 are shown by crosses and labelled S1. The saddle point at the origin is labelled $\mathrm{O}$.

\section{THE MODEL UNDER STUDY}

To demonstrate the existence of this escape mechanism, we take as an example the two-dimensional map introduced by Holmes. ${ }^{14}$ The properties of this map, including the structures both of its CA and of its locally disconnected FBB, are generic for a wide class of maps and flow systems..$^{20,21}$ This fact, and the results of our investigations of escape in other systems, allow us to believe that the mechanism of escape we will reveal is in fact a typical one. The Holmes map is

$$
\begin{aligned}
& x_{n+1}=y_{n} \\
& y_{n+1}=-b x_{n}+d y_{n}-y_{n}^{3}+\xi_{n},
\end{aligned}
$$

where $\xi_{n}$ is zero-mean white Gaussian noise of variance $D$. Due to symmetry, the noise-free system (1) possesses pairs of co-existing attractors for $b=0.2$ and $2.0 \leq d \leq 2.745$ the basins of which are separated by a boundary that may be either smooth or fractal depending on the choice of parameter values. We chose for our studies $b=0.2$ and $d=2.7$, which corresponds to there being two co-existing CAs whose basins are separated by a locally disconnected FBB (see Fig. 1). The fractal dimension of the boundary has been determined numerically $($ dim. $=1.84472)$ by using the uncertainty exponent technique introduced in. ${ }^{22}$ For our chosen parameters, the chaotic attractors in (1) appear as the result of a period-doubling cascade, and each of them consists of two disconnected parts.

\section{BOUNDARY CONDITIONS}

We excited the system (1) with weak noise and collected both the trajectories that include escape paths from one $\mathrm{CA}$ to the other, and the corresponding realisations of noise that induced these transitions. By averaging a few hundred escape trajectories and noise realisations, we obtained the optimal escape path and the corresponding optimal force, which are shown in Fig. 2. The results of this statistical analysis allowed us to determine both the boundary conditions near the CA and the FBB and to demonstrate the uniqueness of the MPEP. It can be seen in particular that the system (1) leaves the CA, falling into a small neighbourhood of the saddle point of period 1 (S1) located between its two disconnected parts and having the multipliers $\rho_{1}=0.118975$ and $\rho_{2}=1.681025$. Its stable manifolds separate the parts of the CA, while the unstable ones belong to the CA. Further, the system makes a few iterations in some small neighbourhood of S1 (initial plateau in Fig. 2(a)) and then moves to the FBB 

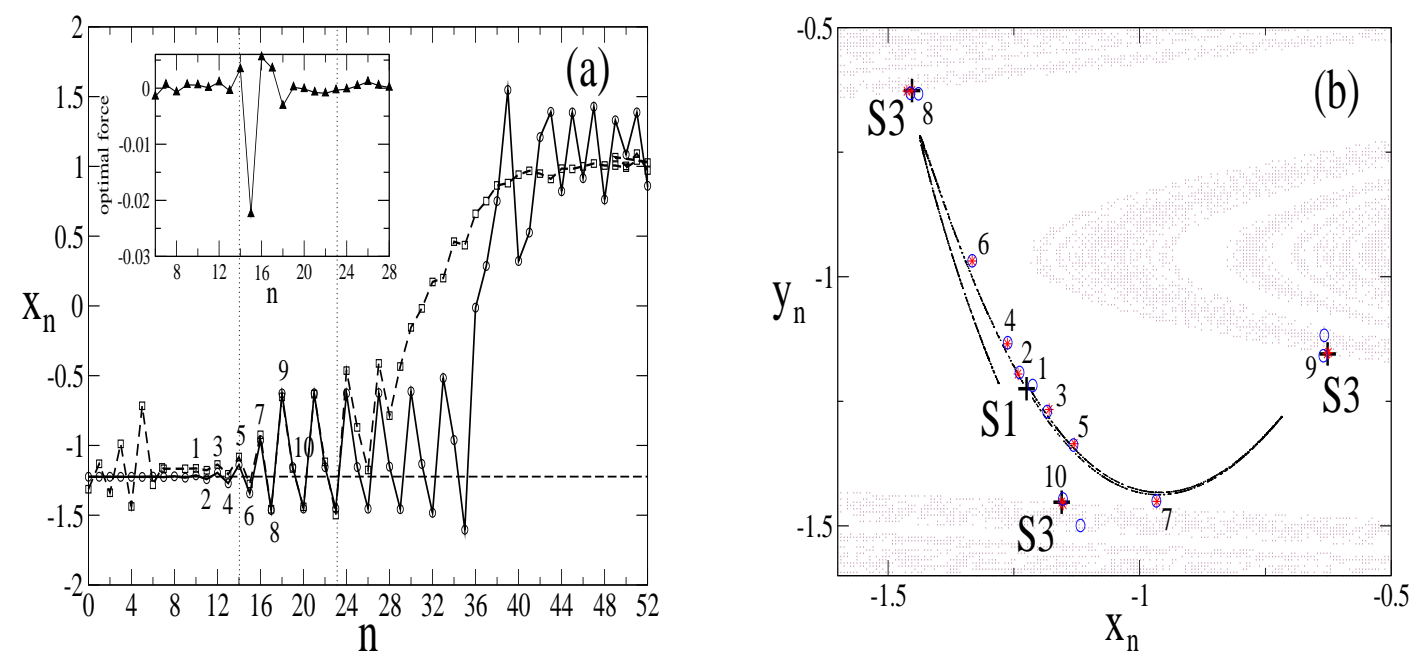

Figure 2. (a) The most probable escape path (dashed line) connecting the CA with the period-3 saddle cycle lying on the fractal boundary, obtained from the numerical simulations with $D=10^{-5}$. The optimal path found by the solution of the boundary-value problem is shown as a solid line. The abscissa of the saddle point S1 shown by the dashed line; (b) a two-dimensional plot of the paths presented in (a) with consecutively numbered points marked as circles (boundary value problem) and stars (obtained from the Monte-Carlo simulation).

in three steps, crossing it at a saddle point of period 3 (S3) with multipliers $\rho_{1}=0.001016$ and $\rho_{2}=7.875512$. Calculations have shown that the S3 for the chosen parameter values lies on the FBB. Moreover, its stable manifold (full black curve in Fig. 1) detaches the open neighborhood, including the attractor, from the FBB itself. The latter fact allows to classify it as an accessible boundary point. ${ }^{15}$ Analysis of the structure of escape paths inside the FB have shown that the homoclinic saddle points play a key role in its formation. In the system (1), we observe an infinite sequence of saddle-node bifurcations of period $3,4,5,6,7 \ldots$, which occur at parameter values $d_{3}<d_{4}<d_{5}<d_{6}<d_{7} \ldots$ and are caused by the sequent tangencies of the stable and unstable manifolds of the saddle point $O$ at origin. The bifurcation diagramm for the few of them presented in Fig. 3(a). The homoclinic orbits appearing as the result of these bifurcations were classified earlier as original saddles and was also shown that their stable and unstable manifolds cross each other in the hierarchical sequence (see Fig. 3(b)). These saddles have the property that their associated eigenvalues of the linearized map are both positive. ${ }^{15}$ To characterize this hierarchical relation between original saddles it is reasonable to introduce a parameter $\mu$ equal to the ratio $\left|\ln \left(\rho_{1}(P)\right)\right| / \ln \left(\rho_{2}(P)\right)$, where $\rho_{1}$ and $\rho_{2}$ are the multipliers of a saddle point $P$. Calculations have shown that, for the original saddles with periods $3,4,5,6,7,8 \ldots$ in $(1)$, the following hierarchical sequence of index $\mu$ values occurs: $\mu_{3}=3.339, \mu_{4}=3.08, \mu_{5}=2.999, \mu_{6}=2.339, \mu_{7}=1.958, \mu_{8}=1.539$. Moreover, the values of index $\mu$ corresponding to the other homoclinic saddle cycles are close to zero. Correspondingly the probability of finding the system in their neighbourhood tends to zero.

These results allows us to formulate generic features of a fluctuational transition through a locally disconnected FBB, as follows: (i) it always occurs through a unique accessible boundary point; and (ii) the original saddles forming the homoclinic structure of the system (1) play a key role in the formation of the paths inside the FBB, and the difference in their local stability defines the hierarchical relationship between them. Thus, we may claim that the complicated structure of escape trajectories, caused by the thin homoclinic structure and their randomness, has in many respects a deterministic nature.

\section{MOST PROBABLE ESCAPE PATH}

An understanding of the escape mechanism allows us to find the MPEP. According to the Hamiltonian theory of fluctuations ${ }^{1-4}$ the MPEP is the path which minimizes the energy

$$
S=\frac{1}{2} \sum_{n=1}^{N} \xi_{n}^{T} \xi_{n}
$$



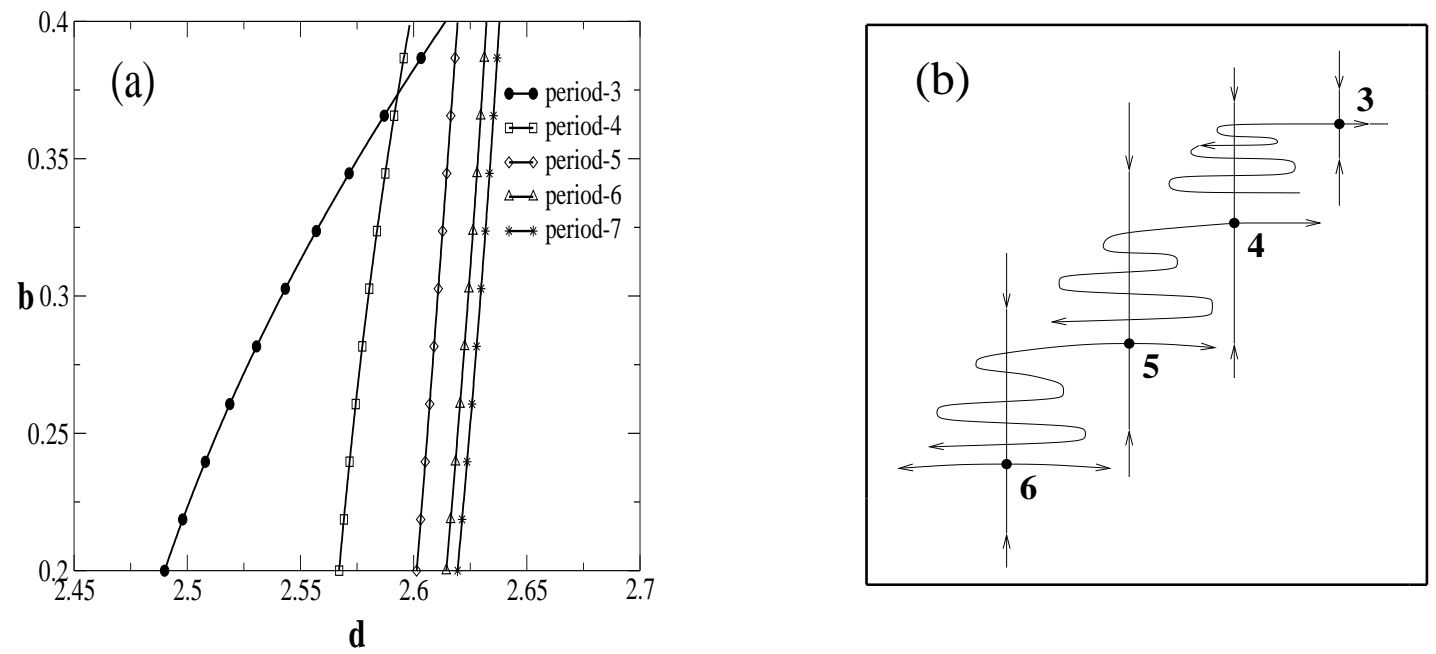

Figure 3. (a) The saddle-node bifurcation lines resulting the appearance of corresponding original saddles; (b) schematic illustration of the intersection between stable and unstable manifolds of original saddles.

of the possible realizations of noise $\left\{\xi_{n}\right\}$ that induce a transition of the system (1) from the CA (with the initial condition on the S1) to the FBB (with the final condition on the accessible orbit S3). The Lagrangian of the corresponding variational problem can be found ${ }^{3}\left(\right.$ cf. Dykman $\left.^{16}\right)$ in the form

$$
L=\frac{1}{2} \sum_{n=1}^{N} \xi_{\mathbf{n}}^{\mathbf{T}} \xi_{\mathbf{n}}+\sum_{n=1}^{N} \lambda_{\mathbf{n}}^{\mathbf{T}}\left(\mathbf{x}_{\mathbf{n}+\mathbf{1}}-\mathbf{f}\left(\mathbf{x}_{\mathbf{n}}\right)-\xi_{\mathbf{n}}\right),
$$

where (1) is taken into account by use of the Lagrange multiplier $\lambda_{n}$. Varying $L$ with respect to $\xi_{n}, \lambda_{n}$, and $x_{n}$, the following area-preserving map is obtained:

$$
\begin{aligned}
x_{n+1} & =y_{n} \\
y_{n+1} & =-b x_{n}+d y_{n}-y_{n}^{3}+\lambda_{n}^{y} \\
\lambda_{n+1}^{x} & =\left(d-3 x_{n+1}^{2}\right) \lambda_{n}^{x} / b-\lambda_{n}^{y} / b \\
\lambda_{n+1}^{y} & =\lambda_{n}^{x}
\end{aligned}
$$

Equations (3) are supplemented by the following boundary conditions:

$$
\lim _{n \rightarrow-\infty} \lambda_{n}^{y}=0, \quad\left(x_{n}^{0}, y_{n}^{0}\right) \in S 1, \quad\left(x_{n}^{1}, y_{n}^{1}\right) \in S 3 .
$$

The MPEP is the minimum-energy heteroclinic trajectory in the phase space of (3) connecting S1 and S3. The solution of this boundary value problem is in general complicated because of the presence of multiple local minima of the energy ${ }^{17}$ induced by the complex geometrical structure of the unstable manifolds of S3 in the phase space of (1) (see e.g. Graham and $\mathrm{Tel}^{19}$ for a discussion). A technique for solving the boundary value problem was introduced recently. ${ }^{18}$ It involves a parameterization of the structure of the multiple local minima by making a proper parameterization of the unstable manifold in the vicinity of the initial conditions. The MPEP found by this method is shown in the Fig. 2. It can be seen from Fig. 2, that the MPEP predicted by the Hamiltonian theory coincides with that obtained using statistical analysis of the escape trajectories in Monte Carlo simulations. Note that no action is required to bring the system to another attractor after it arrives on the accessible orbit on the FBB.

The existence of an almost deterministic mechanism of transition via the FBB itself raises an important question about the effect of noise on this mechanism. To study the influence of noise on the structure of the 


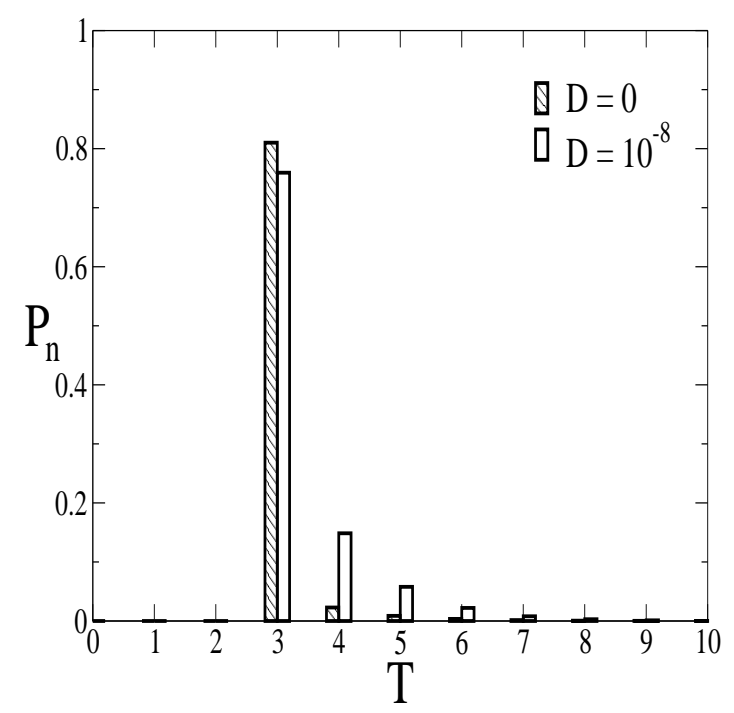

Figure 4. Probabilities of finding a fragment corresponding to the different period- $T$ original saddle cycle in the collected escape trajectories.

escape trajectories inside the FBB we used randomly chosen initial conditions in a very small neighborhood of the accessible point S3 through which the escape occurs (see Fig. 2(b)). By definition of the FBB, any arbitrarily small neighborhood of S3 contains points that belong to the basin of the other attractor. Therefore the system may cross the FBB starting from a very small neighborhood of S3 even in the absence of noise. Collecting all successful escape paths, we calculated the probabilities for the system to pass via small neighborhoods of different original saddle cycles during its escape both in the presence and in the absence of noise. As can be seen from Fig. 4, the corresponding probabilities demonstrate the same hierarchical interrelationship in both cases. This hierarchical interrelationship is determined by the value of index $\mu$ defined above. This structure is robust with respect to the noise-induced perturbations. The addition of noise causes a small broadening of the distribution in Fig. 4 and a small increase of the probability for the system to escape via original saddles of larger period.

\section{CONCLUSIONS}

In conclusion, we have described the mechanism by which noise-induced escape occurs through a locally disconnected FBB. We have found the (unique) most probable escape path from a chaotic attractor to the fractal boundary, using both statistical analyses of fluctuational trajectories, and the Hamiltonian theory of fluctuations. We have shown that the original saddles forming the homoclinic structure play a key role in effecting the transition through the FBB itself. In particular, their local stability defines the hierarchical relationship between the probabilities for the system to pass via small neighborhoods of different original saddle cycles during its escape, both in the presence and in the absence of noise. We emphasize that the escape mechanism we have revealed must be applicable to the broad class of two dimensional maps and flows ${ }^{14,20,21}$ that exhibit the same type of FBB. For instance, one possible application of our results is to the development of an energy-optimal control scheme for the $\mathrm{CO}_{2}$ laser, a discrete model of which demonstrates the type of FBB considered above. ${ }^{23}$

\section{ACKNOWLEDGMENTS}

The authors would like to thank Ulrike Feidel, Suso Kraut and Igor Khovanov for fruitful discussions. The research has been supported by the Engineering and Physical Sciences Research Council (UK).

\section{REFERENCES}

1. R. L. Kautz, "Activation energy for thermally induced escape from a basin of attraction," Phys. Lett. A 125, pp. 315-319, 1987. 
2. P. D. Beale, "Noise-induced escape from attractor in one-dimensional maps," Phys. Rev. A 40, pp. 39984003, 1989.

3. P. Grassberger, "Noise-induced escape from attractors," J. Phys. A: Math. Gen. 22, pp. 3283-3290, 1989.

4. R. Graham, A. Hamm, and T. Tel, "Nonequilibrium potentials for dynamical systems with fractal attractors or repellers," Phys. Rev. Lett. 66, pp. 3089-3092, 1991.

5. J. Guckenheimer and P. Holmes, Nonlinear Oscillations, Dynamical Systems, and Bifurcations of Vector Fields, Springer-Verlag, New-York, 1983.

6. E. Ott, Chaos in Dynamical Systems, Cambridge University Press, Cambridge, 2002.

7. L. Onsager, and S. Machlup, "Fluctuations and irreversible processes," Phys. Rev 91, pp. 1505-1512, 1953.

8. M. I. Dykman, P. V. E. McClintock, V. N. Smelyanskiy, N. D. Stein, and N. G. Stocks, "Optimal paths and the prehistory problem for large fluctuations in noise driven systems," Phys. Rev. Lett. 68, pp. 2718-2721, 1992 .

9. D. G. Luchinsky, R. S. Maier, R. Mannella, P.V.E. McClintock, D. L. Stein, "Experiments on critical phenomena in a noisy exit problem," Phys. Rev. Lett. 79, pp. 3117-3120, 1997.

10. D. G. Luchinsky, "On the nature of large fluctuations in equilibrium systems: observation of an optimal force," J. Phys. A 30, pp. L577-L583, 1997.

11. D. G. Luchinsky and P. V. E. McClintock, "Irreversibility of classical fluctuations studied in analogue electrical circuits" Nature 389, pp. 463-466 1997.

12. M. I. Freidlin and A. D. Wentzel, Random Perturbations in Dynamical Systems, Springer-Verlag, New York, 1984.

13. S.M. Soskin, M. Arrays, R. Mannella, and A.N. Silchenko, "Strong enhancement of the noise-induced escape by non-adiabatic periodic driving due to transient chaos," Phys. Rev. E. 63, pp. 051111-6, 2001.

14. P. Holmes, "A nonlinear oscillator with a strange attractor," Phil. Trans. R. Soc. A292, pp. 419-448 (1979).

15. C. Grebogi, E. Ott, and J.A. Yorke, "Basin boundary metamorphoses:changes in accessible boundary points," Physica D 24, pp. 243-262, 1987.

16. M. I. Dykman, "Large fluctuations andfluctuational transitions in systems driven by colored Gaussian noisea high frequency noise," Phys. Rev. A 42, pp. 2020-2029, 1990.

17. D. G. Luchinsky, S. Beri, R. Mannella, P. V. E. McClintock and I. A. Khovanov, "Optimal fluctuations and the control of chaos," Int. J. Bifurcation and Chaos 12, pp. 583-604, 2002.

18. S. Beri, D. G. Luchinsky, R. Mannella, P. V. E. McClintock, Submitted to Phys. Rev. E.

19. R. Graham and T. Tel, "Existence of a potential for dissipative dynamical systems," Phys. Rev. Lett. 52, pp. 9-12, 1984.

20. M. L. Cartwright and J. E. Littlewood, Ann. Math. 54, pp. 1-19, 1951.

21. S.W. McDonald, C. Grebogi, E. Ott, and J.A. Yorke, "Fractal basin boundaries," Physica D 17 pp. 125-153, 1985.

22. C. Grebogi, S. W. McDonald, E. Ott, and J. A. Yorke, "Final-state sensitivity - an obstruction to predictability," Phys. Lett. A 99 pp. 15-418, 1983.

23. V. N. Chizhevsky, E. V. Grigorieva and S. A. Kashchenko, "Optimal timing for targeting periodic orbits in a loss-driven CO2 laser," Opt. Commun. 133, pp. 189-195, 1997. 